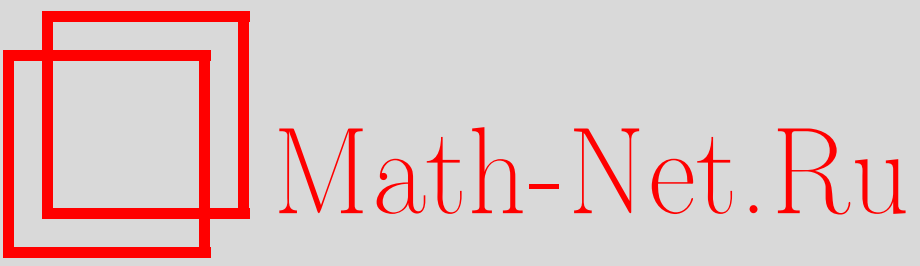

М. Бойти, Ф. Пемпинелли, А. К. Погребков, Б. Принари, К спектральной теории нестационарного уравнения Шредингера с двумерно возмущенным произвольным одномерным потенциалом, ТM $\Phi, 2005$, том 144, номер 2, 257-276

DOI: https://doi.org/10.4213/tmf1851

Использование Общероссийского математического портала Math-Net.Ru подразумевает, что вы прочитали и согласны с пользовательским соглашением

http://www.mathnet.ru/rus/agreement

Параметры загрузки:

IP : 44.207 .124 .84

26 апреля 2023 г., 13:42:21 


\section{К СПЕКТРАЛЬНОЙ ТЕОРИИ НЕСТАЦИОНАРНОГО УРАВНЕНИЯ ШРЕДИНГЕРА С ДВУМЕРНО ВОЗМУШЕННЫМ ПРОИЗВОЛЬНЫМ ОДНОМЕРНЫМ ПОТЕНЦИАЛОМ}

В рамках подхода расширенной резольвенты рассматривается нестационарное уравнение Шредингера, потенциал которого является возмущением произвольного одномерного потенциала посредством убывающей двумерной функции. Приведены соответствующие модификации решений Йоста, опережающих и запаздывающих решений и спектральных данных, а также соотношения между ними.

Ключевые слова: метод обратной задачи рассеяния, резольвентный подход, уравнение Кадомцева-Петвиашвили.

\section{1. ВВЕДЕНИЕ}

Уравнение Кадомцева-Петвиашвили в версии, которая называется КПІ, имеет вид

$$
\left(u_{t}-6 u u_{x_{1}}+u_{x_{1} x_{1} x_{1}}\right)_{x_{1}}=3 u_{x_{2} x_{2}}
$$

и является $(2+1)$-мерным обобшением известного уравнения Кортевега-де Фриза $(\mathrm{Kд \Phi )} \mathrm{[1],} \mathrm{[2].} \mathrm{Как} \mathrm{следствие,} \mathrm{уравнение} \mathrm{КПІ} \mathrm{допускает} \mathrm{решения,} \mathrm{ведушие} \mathrm{себя} \mathrm{при}$ бесконечно удаленных $x$ подобно решениям уравнения КдФ. Например, если $u_{1}\left(t, x_{1}\right)$ удовлетворяет уравнению $\mathrm{K} д \Phi$, то $u\left(t, x_{1}, x_{2}\right)=u_{1}\left(t, x_{1}+\mu x_{2}+3 \mu^{2} t\right)$ является решением уравнения КПІ при произвольной константе $\mu \in \mathbb{R}$. Поэтому естественно рассматривать такие решения уравнения (1.1), которые убывают на бесконечности по всем направлениям, кроме некоторых одномерных лучей, вдоль которых решение ведет себя как $u_{1}$. Хотя уже около тридцати лет известно, что уравнение КПІ интегрируемо [2], его обшая теория далека от завершения. Действительно, в работах [3] задача Коши

*Dipartimento di Fisica, Università di Lecce and Sezione INFN, Lecce, Italy.

E-mail: boiti@le.infn.it, pempi@le.infn.it, prinari@le.infn.it

* Математический институт им. В. А. Стеклова РАН, ул. Губкина 8, 119991, Москва, Россия. E-mail: pogreb@mi.ras.ru 
для КПІ с быстроубываюшими начальными данными была решена с помощью метода обратной задачи рассеяния (МОЗР) на основе спектрального анализа нестационарного оператора Шредингера

$$
\mathcal{L}\left(x, \partial_{x}\right)=i \partial_{x_{2}}+\partial_{x_{1}}^{2}-u(x), \quad x=\left(x_{1}, x_{2}\right),
$$

задаюшего вспомогательную линейную задачу для уравнения КПІ. Однако, как известно, стандартный подход к спектральной теории оператора (1.2), основанный на интегральных уравнениях для решений Йоста, неприменим для потенциалов с одномерным асимптотическим поведением.

В работах [4] был предложен метод расширенной резольвенты как способ построения обобщения МОЗР, позволяющего изучать спектральную теорию операторов с нетривиальным асимптотическим поведением на пространственной бесконечности. Здесь мы рассматриваем случай, когда имеется только одно направление неубывающего поведения, а именно, когда

$$
u(x)=u_{1}\left(x_{1}\right)+u_{2}\left(x_{1}, x_{2}\right),
$$

поэтому без потери обшности мы выбрали константу $\mu=0$ (обший случай восстанавливается за счет галилеевой инвариантности уравнения (1.1)). Спектральная теория для простейшего примера потенциала в (1.2) была развита в работе [5], в которой для уравнения КПІ была рассмотрена задача Коши с начальным условием типа (1.3), причем $u_{1}\left(x_{1}\right)$ являлось значением при $t=0$ односолитонного решения уравнения $\mathrm{K} д \Phi$, а $u_{2}(x)$ - гладкой, вещественной, быстроубывающей функцией на $\left(x_{1}, x_{2}\right)$-плоскости. В работе [5] была рассмотрена прямая задача и с помошью модифицированных интегральных уравнений было показано, что модифицированное решение Йоста в дополнение к стандартному скачку на вешественной оси спектрального параметра $\mathbf{k}$ имеет также скачок на отрезке мнимой оси комплексной $\mathbf{k}$-плоскости. Однако в этой статье некоторые существенные свойства решений Йоста и соотношения между ними были даны без доказательства. В работе [6] задача была полностью решена в рамках подхода расширенной резольвенты для случая чисто односолитонного потенциала $u_{1}$. Здесь мы даем обобшение этих результатов на случай, когда $u_{1}\left(x_{1}\right)$ в $(1.3)$ - произвольный гладкий, вещественный и быстроубывающий по $x_{1}$ одномерный потенциал. Для краткости некоторые результаты приведены здесь без доказательств, которые можно будет найти в [7]. Там же будут выведены характеристические уравнения для спектральных данных и исследованы их свойства.

\section{2. СЛУЧАЙ ОДНОМЕРНОГО ПОТЕНЦИАЛА}

2.1. Основные объекты резольвентного подхода. В этом разделе мы даем краткий обзор основных элементов подхода расширенной резольвенты. Дальнейшие детали заинтересованный читатель может найти в [4]. Пусть $A\left(x, x^{\prime} ; q\right)$ принадлежит пространству $\mathcal{S}^{\prime}$ обобщенных функций умеренного роста от шести вещественных переменных $x=\left(x_{1}, x_{2}\right), x^{\prime}=\left(x_{1}^{\prime}, x_{2}^{\prime}\right)$ и $q=\left(q_{1}, q_{2}\right)$. Мы называем $A(q)$ оператором с ядром 
$A\left(x, x^{\prime} ; q\right) \in \mathcal{S}^{\prime}$. Для операторов $A(q)$ и $B(q)$ с ядрами $A\left(x, x^{\prime} ; q\right)$ и $B\left(x, x^{\prime} ; q\right)$ мы вводим стандартньй закон композиции

$$
(A B)\left(x, x^{\prime} ; q\right)=\int d x^{\prime \prime} A\left(x, x^{\prime \prime} ; q\right) B\left(x^{\prime \prime}, x^{\prime} ; q\right),
$$

если интеграл существует в смысле обобшенных функций. Оператор $A$ может обладать обратным в терминах этой композиции, $A A^{-1}=I$ или $A^{-1} A=I$, где $I$ - единичньй оператор в $\mathcal{S}^{\prime}, I\left(x, x^{\prime} ; q\right)=\delta\left(x-x^{\prime}\right)$, а $\delta(x)=\delta\left(x_{1}\right) \delta\left(x_{2}\right)$ является двумерной $\delta$-функцией. Сушественный для нас подкласс в этом пространстве операторов получается следующим образом. Пусть $\mathcal{A}=\mathcal{A}\left(x, \partial_{x}\right)$ означает дифференциальный оператор с ядром $A\left(x, x^{\prime}\right)=\mathcal{A}\left(x, \partial_{x}\right) \delta\left(x-x^{\prime}\right)$. В дальнейшем мы рассматриваем дифференшиальные операторы, коэффициенты которых являются гладкими функциями от $x$. Мы вводим расширение дифференциальных операторов, т.е. каждому дифференциальному оператору $\mathcal{A}$ мы сопоставляем оператор $A(q)$ с ядром

$$
A\left(x, x^{\prime} ; q\right) \equiv \mathcal{A}\left(x, \partial_{x}+q\right) \delta\left(x-x^{\prime}\right)=e^{-q\left(x-x^{\prime}\right)} A\left(x, x^{\prime}\right),
$$

где $x, x^{\prime}, q \in \mathbb{R}^{2}$ и $q x=q_{1} x_{1}+q_{2} x_{2}$. Такие ядра, очевидно, принадлежат пространству $\mathcal{S}^{\prime}\left(\mathbb{R}^{6}\right)$.

Сопоставим каждому оператору $A(q)$ с ядром $A\left(x, x^{\prime} ; q\right)$ его "хэт-ядро"

$$
\hat{A}\left(x, x^{\prime} ; q\right)=e^{q\left(x-x^{\prime}\right)} A\left(x, x^{\prime} ; q\right) .
$$

Если $A(q)$ - дифференциальный оператор, то это равенство задает соответствие, обратное $(2.2), \hat{A}\left(x, x^{\prime} ; q\right)=A\left(x, x^{\prime}\right)$, и для любого (не обязательно дифференциального) оператора $B(q)$ выполняются следуюшие соотношения:

$$
\begin{aligned}
& \widehat{(A B})\left(x, x^{\prime} ; q\right)=\mathcal{A}\left(x, \partial_{x}\right) \widehat{B}\left(x, x^{\prime} ; q\right), \\
& (\widehat{B A})\left(x, x^{\prime} ; q\right)=\mathcal{A}^{\mathrm{d}}\left(x^{\prime}, \partial_{x^{\prime}}\right) \widehat{B}\left(x, x^{\prime} ; q\right),
\end{aligned}
$$

где $\mathcal{A}^{\mathrm{d}}$ - оператор, дуальньй к $\mathcal{A}$. В дальнейшем для равенств типа (2.4) мы будем использовать обозначения

$$
\widehat{A B}=\overrightarrow{\mathcal{A}} \widehat{B}, \quad \widehat{B A}=\widehat{B} \overleftarrow{\mathcal{A}}
$$

Расширение $L(q)$ оператора $\mathcal{L}\left(x, \partial_{x}\right)$ из (1.2) дается посредством соотношения

$$
L(q)=L_{0}(q)-U,
$$

где в соответствии с $(2.2) \quad L_{0}$ имеет ядро

$$
L_{0}\left(x, x^{\prime} ; q\right)=\left[i\left(\partial_{x_{2}}+q_{2}\right)+\left(\partial_{x_{1}}+q_{1}\right)^{2}\right] \delta\left(x-x^{\prime}\right),
$$

а ядро оператора $U$ равно

$$
U\left(x, x^{\prime} ; q\right)=u(x) \delta\left(x-x^{\prime}\right)
$$


причем функция $u(x)$ всегда полагается вешественной. Основным объектом нашего подхода является (расширенная) резольвента $M(q)$ оператора $L(q)$, которая определяется как оператор, обратный к оператору $L$ :

$$
L M=M L=I .
$$

Мы опускаем здесь для краткости дополнительные условия, гарантирующие единственность расширенной резольвенты как решения уравнений (2.9), ссылаясь, например, на работу [6]. Хэт-ядро (см. (2.3)) расширенной резольвенты “голого” оператора $L_{0}$ задается как

$$
\begin{gathered}
\widehat{M}_{0}\left(x, x^{\prime} ; q\right)=\frac{1}{2 \pi i} \int_{\mathbf{k}_{\Im}=q_{1}} d \mathbf{k}_{\Re}\left[\theta\left(x_{2}-x_{2}^{\prime}\right)-\theta\left(\ell_{2 \Im}(\mathbf{k})-q_{2}\right)\right] \Phi_{0}(x, \mathbf{k}) \Psi_{0}\left(x^{\prime}, \mathbf{k}\right), \\
\text { где } \Phi_{0}(x, \mathbf{k})=e^{-i \ell(\mathbf{k}) x}, \Psi_{0}(x, \mathbf{k})=e^{i \ell(\mathbf{k}) x} \text { и } \\
\ell(\mathbf{k})=\left(\mathbf{k}, \mathbf{k}^{2}\right), \quad \mathbf{k}=\mathbf{k}_{\Re}+i \mathbf{k}_{\Im} \in \mathbb{C}
\end{gathered}
$$

- двухкомпонентный вектор. Мы используем полужирный шрифт для обозначения комплексных переменных. Для любого $\mathbf{k} \in \mathbb{C}$ функции $\Phi_{0}(x, \mathbf{k})$ и $\Psi_{0}(x, \mathbf{k})$ являются решениями нестационарного уравнения Шредингера (1.2) и удовлетворяют свойству сопряжения $\overline{\Phi_{0}(x, \mathbf{k})}=\Psi_{0}(x, \overline{\mathbf{k}})$. Используя обозначения $(2.5)$, равенства $(2.9)$ для случая нулевого потенциала можно записать в виде $\overrightarrow{\mathcal{L}}_{0} \widehat{M}_{0}(q)=\widehat{M}_{0}(q) \overleftarrow{\mathcal{L}}_{0}=I$, которое показывает, что $\widehat{M}_{0}(q)$ - двупараметрическое семейство функций Грина уравнения (1.2) и дуального к нему.

Из (2.10) непосредственно следует, что при $q_{1} \neq 0$

$$
\begin{aligned}
& \frac{\partial \widehat{M}_{0}(q)}{\partial q_{1}}=\frac{i}{\pi} \int_{\mathbf{k}_{\Im}=q_{1}} d \mathbf{k}_{\Re} \overline{\mathbf{k}} \delta\left(\ell_{2 \Im}(\mathbf{k})-q_{2}\right) \Phi_{0}(\mathbf{k}) \otimes \Psi_{0}(\mathbf{k}), \\
& \frac{\partial \widehat{M}_{0}(q)}{\partial q_{2}}=\frac{1}{2 \pi i} \int_{\mathbf{k}_{\Im}=q_{1}} d \mathbf{k}_{\Re} \delta\left(\ell_{2 \Im}(\mathbf{k})-q_{2}\right) \Phi_{0}(\mathbf{k}) \otimes \Psi_{0}(\mathbf{k}),
\end{aligned}
$$

где $\ell_{2} \Im$ - мнимая часть второй компоненты вектора, введенного в $(2.11)$, а прямое произведение определено стандартным образом как оператор с ядром

$$
\left(\Phi_{0}(\mathbf{k}) \otimes \Psi_{0}(\mathbf{k})\right)\left(x, x^{\prime}\right)=\Phi_{0}(x, \mathbf{k}) \Psi_{0}\left(x^{\prime}, \mathbf{k}\right) .
$$

С помощью $M_{0}$ резольвента общего оператора $L$ из (2.6) также может быть определена как решение интегральных уравнений

$$
M=M_{0}+M_{0} U M, \quad M=M_{0}+M U M_{0} .
$$

Снова ссылаясь на работы [4], отметим здесь, что основной инструмент для исследования свойств резольвенты выводится из тождества

$$
M^{\prime}-M=-M^{\prime}\left(L^{\prime}-L\right) M
$$

где $L^{\prime}$ - другой оператор типа (1.2) и $M^{\prime}$ - его резольвента. Это тождество Гильберта (мы называем его так по аналогии со стандартной спектральной теорией операторов) используется для исследования свойств непрерьвности и дифференцируемости резольвенты по $q$-переменным. 
2.2. Резольвентный подход в случае одномерного потенциала. Ниже в этом разделе мы рассмотрим вложение стандартного одномерного преобразования рассеяния, см. [8], в теорию двумерных дифференциальных операторов с одномерным потенциалом. Это дает нам базовьй пример потенциала (1.3) с $u_{2} \equiv 0$. Результаты этого построения будут использованы в следующем разделе, где изучается обший случай $u_{2} \neq 0$. Итак, мы рассматриваем здесь расширенный дифференциальный оператор

$$
L_{1}(q)=L_{0}(q)-U_{1}, \quad U_{1}\left(x, x^{\prime} ; q\right)=u_{1}\left(x_{1}\right) \delta\left(x-x^{\prime}\right),
$$

где $L_{0}(q)$ определен в (2.7). Для решений Йоста нестационарного уравнения Шредингера и дуального к нему мы используем обозначения

$$
\begin{gathered}
\varphi(x, \mathbf{k})=e^{-i \mathbf{k}^{2} x_{2}} \Phi_{1}\left(x_{1}, \mathbf{k}\right)=e^{-i \mathbf{k} x_{1}-i \mathbf{k}^{2} x_{2}} \chi_{1}\left(x_{1}, \mathbf{k}\right), \\
\psi(x, \mathbf{k})=e^{i \mathbf{k}^{2} x_{2}} \Psi_{1}\left(x_{1}, \mathbf{k}\right)=e^{i \mathbf{k} x_{1}+i \mathbf{k}^{2} x_{2}} \xi_{1}\left(x_{1}, \mathbf{k}\right),
\end{gathered}
$$

соответственно, где $\Phi_{1}\left(x_{1}, \mathbf{k}\right)$ и $\Psi_{1}\left(x_{1}, \mathbf{k}\right)$ являются решениями Йоста одномерного оператора Штурма-Лиувилля, а именно, $\chi_{1}$ определено интегральным уравнением

$$
\chi_{1}\left(x_{1}, \mathbf{k}\right)=1+\int_{-\mathbf{k}_{\Im} \infty}^{x_{1}} d y_{1} \frac{e^{2 i \mathbf{k}\left(x_{1}-y_{1}\right)}-1}{2 i \mathbf{k}} u_{1}\left(y_{1}\right) \chi_{1}\left(y_{1}, \mathbf{k}\right),
$$

и аналогичное уравнение определяет $\xi_{1}\left(x_{1}, \mathbf{k}\right)$. Функции $\varphi(x, \mathbf{k})$ и $\psi(x, \mathbf{k})$, равно как и их предельные значения на вешественной оси, $\varphi^{ \pm}(x, k)=\varphi(x, k \pm i 0)$ и $\psi^{ \pm}(x, k)=$ $\psi(x, k \pm i 0)$, удовлетворяют свойствам сопряжения:

$$
\overline{\varphi(x, \mathbf{k})}=\psi(x, \overline{\mathbf{k}}), \quad \mathbf{k} \in \mathbb{C} ; \quad \overline{\varphi^{ \pm}(x, k)}=\psi^{\mp}(x, k), \quad k \in \mathbb{R},
$$

а также соотношениям ортогональности и полноты:

$$
\begin{gathered}
\frac{1}{2 \pi} \int d x_{1} \psi(x, \mathbf{k}+p) \varphi(x, \mathbf{k})=\frac{\delta(p)}{t(\mathbf{k})}, \quad p \in \mathbb{R}, \quad \mathbf{k} \in \mathbb{C}, \\
\int d x_{1} \psi\left(x, i \varkappa_{j}\right) \varphi(x, \mathbf{k})=\int d x_{1} \psi(x, \mathbf{k}) \varphi\left(x, i \varkappa_{j}\right)=0, \quad\left|\mathbf{k}_{\Im}\right|<\varkappa_{j}, \\
\int d x_{1} \psi\left(x, i \varkappa_{j}\right) \varphi\left(x, i \varkappa_{j^{\prime}}\right)=\frac{i \delta_{j, j^{\prime}}}{t_{j}}, \\
\frac{1}{2 \pi} \int_{x_{2}^{\prime}=x_{2}} d \mathbf{k}_{\Re} t(\mathbf{k}) \varphi(x, \mathbf{k}) \psi\left(x^{\prime}, \mathbf{k}\right)- \\
-\left.i \sum_{j=1}^{N} t_{j} \theta\left(\varkappa_{j}-\left|\mathbf{k}_{\Im}\right|\right) \varphi\left(x, i \varkappa_{j}\right) \psi\left(x^{\prime}, i \varkappa_{j}\right)\right|_{x_{2}^{\prime}=x_{2}}=\delta\left(x_{1}-x_{1}^{\prime}\right),
\end{gathered}
$$

где $t(\mathbf{k})$ - коэффишиент прохождения одномерной задачи с полюсами в точках $\pm i \varkappa_{j}$, $j=1, \ldots, N$ (для определенности мы полагаем $\varkappa_{j}>0$ для всех $j$ ). Обозначим вычеты функции $t(\mathbf{k})$ в верхней полуплоскости как

$$
t_{j}=\underset{\mathbf{k}=i \varkappa_{j}}{\operatorname{res}} t(\mathbf{k}) .
$$


Другой набор дискретных спектральных данных одномерной задачи дается коэффициентами $b_{j}$, определенными одним из следующих равенств:

$$
\Phi_{1}\left(x_{1}, i \varkappa_{j}\right)=b_{j} \Phi_{1}\left(x_{1},-i \varkappa_{j}\right), \quad \Psi_{1}\left(x_{1},-i \varkappa_{j}\right)=b_{j} \Psi_{1}\left(x_{1}, i \varkappa_{j}\right) .
$$

Эти коэффициенты вещественны, отличны от нуля, и $\operatorname{sgn}\left(i t_{j} b_{j}\right)=-1$. Из формулы $(2.20)$ следует, что

$$
\varphi\left(x, i \varkappa_{j}\right)=b_{j} \varphi\left(x,-i \varkappa_{j}\right), \quad \varphi\left(x, i \varkappa_{j}\right)=b_{j} \overline{\psi\left(x, i \varkappa_{j}\right)} .
$$

В силу (2.24) легко проверить (ср. с (2.3)), что хэт-ядро резольвенты $M_{1}(q)$ двумерного оператора $L_{1}(q)$ в терминах предыдущих обозначений дается равенством

$$
\begin{aligned}
\widehat{M}_{1}\left(x, x^{\prime} ; q\right)= & \frac{1}{2 \pi i} \int_{\mathbf{k}_{\Im}=q_{1}} d \mathbf{k}_{\Re}\left[\theta\left(x_{2}-x_{2}^{\prime}\right)-\theta\left(2 \mathbf{k}_{\Re} \mathbf{k}_{\Im}-q_{2}\right)\right] t(\mathbf{k}) \varphi(x, \mathbf{k}) \psi\left(x^{\prime}, \mathbf{k}\right)- \\
& -\sum_{j} \theta\left(\varkappa_{j}^{2}-q_{1}^{2}\right) t_{j}\left[\theta\left(x_{2}-x_{2}^{\prime}\right)-\theta\left(-q_{2}\right)\right] \varphi\left(x, i \varkappa_{j}\right) \psi\left(x^{\prime}, i \varkappa_{j}\right),
\end{aligned}
$$

т.е. в обозначениях $(2.5)$

$$
\overrightarrow{\mathcal{L}}_{1} \widehat{M}_{1}(q)=\widehat{M}_{1}(q) \overleftarrow{\mathcal{L}}_{1}=I
$$

что эквивалентно (2.9). Более того, возврашаясь к ядру $M_{1}\left(x, x^{\prime} ; q\right)$ и используя свойства одномерных решений Йоста и определения (2.18), мы получаем, что $M_{1}\left(x, x^{\prime} ; q\right) \in \mathcal{S}^{\prime}$.

Поведение $\widehat{M}_{1}\left(x, x^{\prime} ; q\right)$ по переменным $q$ определяется комбинацией $\theta$-функций и поведением $t(\mathbf{k})$ в полюсах. Вводя в подынтегральное выражение члены, компенсирующие эти полюсные особенности, мы разлагаем резольвенту в сумму

$$
\widehat{M}_{1}(q)=\widehat{M}_{1, \mathrm{reg}}(q)+\sum_{j} \Gamma_{j}(q) \varphi\left(i \varkappa_{j}\right) \otimes \psi\left(i \varkappa_{j}\right),
$$

где $\widehat{M}_{1, \mathrm{reg}}(q)$ - регулярная функция от $q$, и $\Gamma_{j}(q)$ суть не зависящие от $x$ функции,

$$
\Gamma_{j}(q)=\frac{t_{j} \operatorname{sgn} q_{1}}{2 \pi i} \ln \frac{q_{2}+2 i q_{1}\left(q_{1}-\varkappa_{j}\right)}{q_{2}+2 i q_{1}\left(q_{1}+\varkappa_{j}\right)}, j=1, \ldots, N,
$$

где логарифм имеет разрез по отрищательной части вещественной оси своего аргумента. Это доказывает, что расширенная резольвента в случае одномерного потенциала имеет логарифмические особенности в точках $q=\left( \pm \varkappa_{j}, 0\right)$ и разрыв при $q_{2}=0$. Для скачка резольвенты при $q_{2}=0$ мы получаем из (2.28), что

$$
\widehat{M}_{1}\left(q_{1},+0\right)-\widehat{M}_{1}\left(q_{1},-0\right)=-\sum_{j} t_{j} \theta\left(\varkappa_{j}-\left|q_{1}\right|\right) \varphi\left(i \varkappa_{j}\right) \otimes \psi\left(i \varkappa_{j}\right) .
$$

Для всех остальных значений $q$ резольвента (2.28) имеет производные по $q$ вида (2.12), (2.13). Например, справедливо равенство

$$
\frac{\partial \widehat{M}_{1}(q)}{\partial q_{2}}=\frac{1}{2 \pi i} \int_{\mathbf{k}_{\Im}=q_{1}} d \mathbf{k}_{\Re} \delta\left(\ell_{2 \Im}(\mathbf{k})-q_{2}\right) t(\mathbf{k}) \varphi(\mathbf{k}) \otimes \psi(\mathbf{k}),
$$


которое в окрестности точки $q_{1}=0$ следует понимать в смысле обобщенных функций. Функция Грина $\mathcal{G}_{1}\left(x, x^{\prime}, \mathbf{k}\right)$ уравнения $(1.2)$ вводится посредством редукции

$$
\mathcal{G}_{1}(\mathbf{k})=\left.\widehat{M}_{1}(q)\right|_{q=\ell_{\Im}(\mathbf{k})},
$$

см. (2.11), такой же, как и в случае убывающего потенциала [6]. Тогда из (2.28) мы получаем билинейное представление

$$
\begin{aligned}
\mathcal{G}_{1}\left(x, x^{\prime}, \mathbf{k}\right)= & \frac{1}{2 \pi i} \int d \alpha\left[\theta\left(x_{2}-x_{2}^{\prime}\right)-\theta\left(\mathbf{k}_{\Im}\left(\alpha-\mathbf{k}_{\Re}\right)\right)\right] t\left(\alpha+i \mathbf{k}_{\Im}\right) \times \\
& \times \varphi\left(x, \alpha+i \mathbf{k}_{\Im}\right) \psi\left(x^{\prime}, \alpha+i \mathbf{k}_{\Im}\right)- \\
& -\sum_{j} t_{j} \theta\left(\varkappa_{j}-\left|\mathbf{k}_{\Im}\right|\right)\left[\theta\left(x_{2}-x_{2}^{\prime}\right)-\theta\left(-\mathbf{k}_{\Re} \mathbf{k}_{\Im}\right)\right] \varphi\left(x, i \varkappa_{j}\right) \psi\left(x^{\prime}, i \varkappa_{j}\right),
\end{aligned}
$$

которое обобшает на случай произвольного потенциала $u_{1}$ функцию Грина, использованную в [5] и [6] для чисто односолитонного потенциала. Эта функция Грина удовлетворяет свойству сопряжения $\overline{\mathcal{G}_{1}\left(x, x^{\prime}, \mathbf{k}\right)}=\mathcal{G}_{1}\left(x^{\prime}, x, \overline{\mathbf{k}}\right)$, а произведение $g_{1}\left(x, x^{\prime}, \mathbf{k}\right)=$ $e^{i \ell(\mathbf{k})\left(x-x^{\prime}\right)} \mathcal{G}_{1}\left(x, x^{\prime}, \mathbf{k}\right)$ убывает при $\mathbf{k} \rightarrow \infty$. Принимая во внимание, что резольвента удовлетворяет (2.29), мы получаем, что функция Грина удовлетворяет интегральным уравнениям

$$
\mathcal{G}_{1}(\mathbf{k})=\mathcal{G}_{0}(\mathbf{k})+\mathcal{G}_{0}(\mathbf{k}) U_{1} \mathcal{G}_{1}(\mathbf{k}), \quad \mathcal{G}_{1}(\mathbf{k})=\mathcal{G}_{0}(\mathbf{k})+\mathcal{G}_{1}(\mathbf{k}) U_{1} \mathcal{G}_{0}(\mathbf{k}),
$$

она аналитична при $\mathbf{k}_{\Re} \mathbf{k}_{\Im} \neq 0$, и в этой области мы имеем

$$
\frac{\partial \mathcal{G}_{1}(\mathbf{k})}{\partial \mathbf{k}_{\Im}}=\frac{\operatorname{sgn} \mathbf{k}_{\Im}}{2 \pi} t(\mathbf{k}) \varphi(\mathbf{k}) \otimes \psi(\mathbf{k}) .
$$

В силу (2.34) можно записать разложение для функции Грина, аналогичное разложению (2.30):

$$
\mathcal{G}_{1}(\mathbf{k})=\mathcal{G}_{1, \mathrm{reg}}(\mathbf{k})+\sum_{j} \gamma_{j}(\mathbf{k}) \varphi\left(i \varkappa_{j}\right) \otimes \psi\left(i \varkappa_{j}\right)
$$

где в соответствии с (2.31)

$$
\gamma_{j}(\mathbf{k})=\Gamma_{j}\left(\ell_{\Im}(\mathbf{k})\right) \equiv \frac{t_{j} \operatorname{sgn} \mathbf{k}_{\Im}}{2 \pi i} \ln \frac{\mathbf{k}-i \varkappa_{j}}{\mathbf{k}+i \varkappa_{j}} .
$$

Это доказывает, что в дополнение к стандартному скачку на вешественной оси функция Грина имеет логарифмические особенности в точках $\mathbf{k}= \pm i \varkappa_{j}$ и разрыв на мнимой оси при $\left|\mathbf{k}_{\Im}\right|<\max _{j} \varkappa_{j}$, что обобшает результаты, полученные в [5] для случая односолитонного потенциала $u_{1}$.

В [4] мы показали, что в случае убываюшего потенциала сами решения Йоста являются результатом специального транкирования и редукций функций Грина. Однако в применении к введенным выше решениям Йоста $\varphi$ и $\psi$ эти процедуры приводят, как 
и следовало ожидать, к расходящимся выражениям. Они могут быть регуляризованы при $\mathbf{k}_{\Im} \neq 0$ посредством следующей предельной процедуры:

$$
\begin{aligned}
& \varphi(x, \mathbf{k})=\lim _{\varepsilon \rightarrow+0} \int d x^{\prime}\left(\mathcal{G}_{1}(\mathbf{k}) \overleftarrow{\mathcal{L}}_{0}\right)\left(x, x^{\prime}\right) e^{-i \ell(\mathbf{k}) x^{\prime}-i \varepsilon x_{2}^{\prime}} \\
& \psi(x, \mathbf{k})=\lim _{\varepsilon \rightarrow+0} \int d x^{\prime} e^{i \ell(\mathbf{k}) x^{\prime}+i \varepsilon x_{2}^{\prime}}\left(\overrightarrow{\mathcal{L}}_{0} \mathcal{G}_{1}(\mathbf{k})\right)\left(x^{\prime}, x\right)
\end{aligned}
$$

которая дает тождества при подстановке $\mathcal{G}_{1}(\mathbf{k})$ из формулы $(2.35)$.

Коэффициент прохождения, функция Грина и решения Йоста разрывны на вешественной оси $\mathbf{k}$. В дальнейшем мы используем обозначения

$$
\begin{aligned}
t^{ \pm}(k) & =\lim _{\mathbf{k}_{\Im} \rightarrow \pm 0} t\left(k+i \mathbf{k}_{\Im}\right), \quad k \in \mathbb{R}, \\
\varphi^{ \pm}(k) & =\lim _{\mathbf{k}_{\Im} \rightarrow \pm 0} \varphi\left(k+i \mathbf{k}_{\Im}\right), \quad \psi^{ \pm}(k)=\lim _{\mathbf{k}_{\Im} \rightarrow \pm 0} \psi\left(k+i \mathbf{k}_{\Im}\right), \\
\mathcal{G}_{1}^{ \pm}(k) & =\lim _{\mathbf{k}_{\Im} \rightarrow \pm 0} \mathcal{G}_{1}\left(k+i \mathbf{k}_{\Im}\right)
\end{aligned}
$$

для их граничных значений. По определению, $t^{ \pm}(k), \varphi^{ \pm}(k)$ и $\psi^{ \pm}(k)$ - конечные гладкие функции от $k$, в то время как граничное значение функции Грина разрывно при $k=0$, как следует из (2.35).

Вследствие $(2.28)$ резольвента $M_{1}(q)$ разрывна при $q=0$ и $q_{2}=0$. Рассмотрим сначала предел $q_{2} \rightarrow 0$, когда $q_{1} \neq 0$. По формуле (2.34) мы получаем

$$
\lim _{q_{2} \rightarrow \pm 0 \mathbf{k}_{\Im}}\left(\left.\widehat{M}_{1}(q)\right|_{q_{1}=\mathbf{k}_{\Im}}\right)=\mathcal{G}_{1}\left( \pm 0+i \mathbf{k}_{\Im}\right)
$$

и для разрыва функции Грина имеем из (2.32)

$$
\mathcal{G}_{1}\left(+0+i \mathbf{k}_{\Im}\right)-\mathcal{G}_{1}\left(-0+i \mathbf{k}_{\Im}\right)=-\operatorname{sgn} \mathbf{k}_{\Im} \sum_{j} t_{j} \theta\left(\varkappa_{j}-\left|\mathbf{k}_{\Im}\right|\right) \varphi\left(i \varkappa_{j}\right) \otimes \psi\left(i \varkappa_{j}\right)
$$

Разрывность резольвенты $M_{1}(q)$ по $q_{2}$ в нуле, когда $q_{1}=0$, приводит к введению опережаюшей и запаздываюшей функций Грина подобно случаю убываюшего потенциала, т.е. мы полагаем, что

$$
\mathcal{G}_{1, \pm}\left(x, x^{\prime}\right)=\lim _{q_{2} \rightarrow \pm 0} \lim _{q_{1} \rightarrow 0} \widehat{M}_{1}\left(x, x^{\prime} ; q\right)
$$

где предел $q_{1} \rightarrow 0$ не зависит от знака. Тогда с учетом (2.28) мы получаем следуюшее билинейное представление для этих функций Грина в терминах решений Йоста на вещественной оси:

$$
\begin{aligned}
\mathcal{G}_{1, \pm}\left(x, x^{\prime}\right)= & \pm \theta\left( \pm\left(x_{2}-x_{2}^{\prime}\right)\right)\left(\frac{1}{2 \pi i} \int d \alpha t^{\sigma}(\alpha) \varphi^{\sigma}(x, \alpha) \psi^{\sigma}\left(x^{\prime}, \alpha\right)-\right. \\
& \left.-\sum_{j} t_{j} \varphi\left(x, i \varkappa_{j}\right) \psi\left(x^{\prime}, i \varkappa_{j}\right)\right)
\end{aligned}
$$


где $\sigma=+,-$, и для граничных значений решений Йоста и $t(\mathbf{k})$ были использованы обозначения (2.42). Понятно, что опережающая и запаздывающая функции Грина не зависят от знака $\sigma$ в $(2.46)$ и удовлетворяют свойству сопряжения $\overline{\mathcal{G}_{1, \pm}\left(x, x^{\prime}\right)}=\mathcal{G}_{1, \mp}\left(x^{\prime}, x\right)$. Опережаюшее и запаздываюшее решения уравнения (1.2) определяются так же, как в случае убываюшего потенциала, т. е.

$$
\begin{aligned}
& \varphi_{ \pm}(x, k)=\int d x^{\prime}\left(\mathcal{G}_{1, \pm} \overleftarrow{\mathcal{L}}_{0}\right)\left(x, x^{\prime}\right) e^{-i \ell(k) x^{\prime}} \\
& \psi_{ \pm}(x, k)=\int d x^{\prime} e^{i \ell(k) x^{\prime}}\left(\overrightarrow{\mathcal{L}}_{0} \mathcal{G}_{1, \pm}\right)\left(x^{\prime}, x\right)
\end{aligned}
$$

Используя обозначения (2.42), мы получаем по формулам (2.35) и (2.46)

$$
\begin{aligned}
\mathcal{G}_{1}^{\sigma}(k)-\mathcal{G}_{1, \pm}= & \frac{\mp 1}{2 \pi i} \int d \alpha \theta( \pm \sigma(\alpha-k)) t^{\sigma}(\alpha) \varphi^{\sigma}(\alpha) \otimes \psi^{\sigma}(\alpha) \pm \\
& \pm \theta(\mp \sigma k) \sum_{j} t_{j} \varphi\left(i \varkappa_{j}\right) \otimes \psi\left(i \varkappa_{j}\right), \quad \sigma=+,-
\end{aligned}
$$

Опережаюшее и запаздываюшее решения и предельные значения решений Йоста на вешественной оси связаны друг с другом посредством соотношений

$$
\varphi_{ \pm}(k)=\int d p \varphi^{\sigma}(p) \overline{r_{ \pm}^{-\sigma}(p, k)}, \quad t^{\sigma}(k) \varphi^{\sigma}(k)=\int d p \varphi_{ \pm}(p) r_{ \pm}^{\sigma}(p, k),
$$

где

$$
r_{ \pm}^{\sigma}(p, k)=\delta(k-p)[\theta( \pm \sigma k)+\theta(\mp \sigma k) t(\sigma k)]+\theta(\mp \sigma k) \delta(k+p) r^{\sigma}(k)
$$

и $r^{ \pm}(k)$ являются стандартными (левым и правым) коэффициентами отражения (см. формулу (2.53) ниже). Аналогичные соотношения для дуальных решений следуют из (2.20) и свойства сопряжения

$$
\overline{\varphi_{ \pm}(k)}=\psi_{\mp}(k) .
$$

В стандартной теории уравнения Штурма-Лиувилля опережающие и запаздывающие решения не используются, в отличие от случая нестационарного уравнения Шредингера. Здесь мы ввели их для удобства дальнейших ссылок. Стандартные (см. [8]) соотношения между граничными значениями решений Йоста на вещественной оси следуют из подстановки первого уравнения в (2.50) во второе:

$$
t^{\sigma}(k) \varphi^{\sigma}(k)=\int d p \varphi^{-\sigma}(p) f^{-\sigma}(p, k), \quad \sigma=+,-,
$$

где мы ввели спектральные данные

$$
f^{-\sigma}\left(k, k^{\prime}\right)=\int d k^{\prime \prime} \overline{r_{ \pm}^{\sigma}\left(k^{\prime \prime}, k\right)} r_{ \pm}^{\sigma}\left(k^{\prime \prime}, k^{\prime}\right) \equiv \delta\left(k-k^{\prime}\right)+\delta\left(k+k^{\prime}\right) r^{-\sigma}(k)
$$

и использовали (2.51) в последнем равенстве. 


\section{3. МЕТОД ОБРАТНОЙ ЗАДАЧИ РАССЕЯНИЯ НАД НЕТРИВИАЛЬНЫМ ФОНОМ: ДВУМЕРНОЕ ВОЗМУЩЕНИЕ ОДНОМЕРНОГО ПОТЕНЦИАЛА}

3.1. Резольвента. Теперь мы приступаем к изучению спектральных свойств оператора $\mathcal{L}(1.2)$ с потеншиалом, данным в (1.3). Расширение $L(q)$ определено в $(2.6)$, а обратньй к $L(q)$ оператор, т. е. резольвенту $M(q)$, мы задаем одним из интегральных уравнений

$$
M(q)=M_{1}(q)+M_{1}(q) U_{2} M(q), \quad M(q)=M_{1}(q)+M(q) U_{2} M_{1}(q),
$$

где $U_{2}\left(x, x^{\prime} ; q\right)=u_{2}(x) \delta\left(x-x^{\prime}\right)$. Мы полагаем $u_{2}(x)$ вешественной, гладкой и быстроубывающей функцией по обеим переменным $\left(x_{1}, x_{2}\right)$. Более того, мы полагаем ее "малой" в том смысле, что решения $M\left(x, x^{\prime} ; q\right)$ обоих уравнений в $(3.1)$ сушествуют в $\mathcal{S}^{\prime}\left(\mathbb{R}^{6}\right)$ и совпадают. Тогда из (3.1) очевидно следует, что резольвента удовлетворяет (2.9). Ее свойства по отношению к переменным $q$ наследуют соответствуюшие свойства резольвенты $M_{1}(q)$. В частности, $M(q)$ - непрерывная функция от $q$, когда $q \neq 0$ и $q_{2} \neq 0$.

Как уже отмечалось, для исследования свойств резольвенты мы используем тождество Гильберта (2.15), которое запишем в виде

$$
M\left(q^{\prime}\right)-M(q)=M\left(q^{\prime}\right) L_{1}\left(q^{\prime}\right)\left(M_{1}\left(q^{\prime}\right)-M_{1}(q)\right) L_{1}(q) M(q),
$$

так что для производных хэт-ядра (2.3) резольвенты мы получаем

$$
\frac{\partial \widehat{M}(q)}{\partial q_{j}}=\widehat{M}(q) \overleftarrow{\mathcal{L}}_{1} \frac{\partial \widehat{M}_{1}(q)}{\partial q_{j}} \overrightarrow{\mathcal{L}}_{1} \widehat{M}(q), \quad j=1,2, \quad q_{2} \neq 0
$$

Используя в правой части этого равенства производную (2.33), мы приходим к равенствам

$$
\begin{aligned}
& \frac{\partial \widehat{M}(q)}{\partial q_{1}}=\frac{i}{\pi} \int_{\mathbf{k}_{\Im}=q_{1}} d \mathbf{k}_{\Re} \overline{\mathbf{k}} \delta\left(\ell_{2 \Im}(\mathbf{k})-q_{2}\right) t(\mathbf{k}) \Phi(\mathbf{k}) \otimes \Psi(\mathbf{k}), \\
& \frac{\partial \widehat{M}(q)}{\partial q_{2}}=\frac{1}{2 \pi i} \int_{\mathbf{k}_{\Im}=q_{1}} d \mathbf{k}_{\Re} \delta\left(\ell_{2 \Im}(\mathbf{k})-q_{2}\right) t(\mathbf{k}) \Phi(\mathbf{k}) \otimes \Psi(\mathbf{k}),
\end{aligned}
$$

см. (2.11), справедливым при $q_{2} \neq 0$, где мы положили

$$
\begin{aligned}
\Phi(x, \mathbf{k}) & =\int d x^{\prime}\left(\mathcal{L}_{1}^{\mathrm{d}}\left(x^{\prime}, \partial_{x^{\prime}}\right) \mathcal{G}\left(x, x^{\prime}, \mathbf{k}\right)\right) \varphi\left(x^{\prime}, \mathbf{k}\right), \\
\Psi\left(x^{\prime}, \mathbf{k}\right) & =\int d x \psi(x, \mathbf{k}) \mathcal{L}_{1}\left(x, \partial_{x}\right) \mathcal{G}\left(x, x^{\prime}, \mathbf{k}\right)
\end{aligned}
$$

$\left(\mathcal{L}_{1}^{\mathrm{d}}\right.$ - оператор, дуальный к $\left.\mathcal{L}_{1}\right)$, и где функция Грина $\mathcal{G}\left(x, x^{\prime}, \mathbf{k}\right)$ определена как специальное значение самой резольвенты (ср. с (2.34)),

$$
\mathcal{G}\left(x, x^{\prime}, \mathbf{k}\right)=\left.\widehat{M}\left(x, x^{\prime} ; q\right)\right|_{q=\ell_{\Im}(\mathbf{k})} \equiv \widehat{M}\left(x, x^{\prime} ; \mathbf{k}_{\Im}, 2 \mathbf{k}_{\Im} \mathbf{k}_{\Re}\right) .
$$


В дальнейшем мы рассматриваем функцию $\mathcal{G}\left(x, x^{\prime}, \mathbf{k}\right)$ как ядро оператора $\mathcal{G}(\mathbf{k})$, а функции $\Phi(x, \mathbf{k})$ и $\Psi\left(x^{\prime}, \mathbf{k}\right)$ - как "вектор" $\Phi(\mathbf{k})$ и “ковектор" $\Psi(\mathbf{k})$ и для краткости записьваем уравнения (3.5)-(3.7), опуская зависимость от $x, x^{\prime}$, т. е. как

$$
\begin{aligned}
& \Phi(\mathbf{k})=\mathcal{G}(\mathbf{k}) \overleftarrow{\mathcal{L}}_{1} \varphi(\mathbf{k}), \quad \Psi(\mathbf{k})=\psi(\mathbf{k}) \overrightarrow{\mathcal{L}}_{1} \mathcal{G}(\mathbf{k}) \\
& \mathcal{G}(\mathbf{k})=\left.\widehat{M}(q)\right|_{q=\ell_{\Im}(\mathbf{k})}
\end{aligned}
$$

Принимая во внимание, что $\mathcal{L}=\mathcal{L}_{1}-U_{2}$, можно проверить что $\mathcal{G}(\mathbf{k})$ действительно удовлетворяет дифференциальным уравнениям $\overrightarrow{\mathcal{L}} \mathcal{G}(\mathbf{k})=\mathcal{G}(\mathbf{k}) \overleftarrow{\mathcal{L}}=I$, как следует из $(2.9)$ и (2.4). Тогда по формуле (3.8) получаем $\overrightarrow{\mathcal{L}} \Phi(\mathbf{k})=0=\Psi(\mathbf{k}) \overleftarrow{\mathcal{L}}, \mathbf{k} \in \mathbb{C}$, т. е. функции $\Phi(x, \mathbf{k})$ и $\Psi(x, \mathbf{k})$, введенные в $(3.5),(3.6)$, являются решениями Йоста, соответственно, оператора (1.2) и дуального к нему. Заметим, что эти определения уже не нуждаются ни в какой регуляризации типа (2.40), (2.41).

Резольвента $M(q)$, как и $M_{1}(q)$, разрывна при $q=0$ и $q_{2}=0$. В случае, когда сначала выполняется предельный переход $q_{1} \rightarrow 0$, мы вводим по аналогии с (2.45) опережающую и запаздываюшую функции Грина посредством соотношений

$$
\mathcal{G}_{ \pm}\left(x, x^{\prime}\right)=\lim _{q_{2} \rightarrow \pm 0} \lim _{q_{1} \rightarrow 0} \widehat{M}\left(x, x^{\prime} ; q\right) .
$$

$\mathrm{C}$ другой стороны, если $q_{1} \neq 0$, то по (3.9) предельные значения резольвенты выражаются (ср. (2.43)) через предельные значения функции Грина на мнимой оси:

$$
\lim _{q_{2} \rightarrow \pm 0 \mathbf{k}_{\Im}}\left(\left.\widehat{M}(q)\right|_{q_{1}=\mathbf{k}_{\Im}}\right)=\mathcal{G}\left( \pm 0+i \mathbf{k}_{\Im}\right) .
$$

3.2. Скачок функции Грина на мнимой оси. В силу (3.1) и определения (3.9) функция Грина удовлетворяет интегральным уравнениям

$$
\mathcal{G}(\mathbf{k})=\mathcal{G}_{1}(\mathbf{k})+\mathcal{G}_{1}(\mathbf{k}) U_{2} \mathcal{G}(\mathbf{k}), \quad \mathcal{G}(\mathbf{k})=\mathcal{G}_{1}(\mathbf{k})+\mathcal{G}(\mathbf{k}) U_{2} \mathcal{G}_{1}(\mathbf{k})
$$

и свойству сопряжения

$$
\overline{\mathcal{G}\left(x, x^{\prime}, \mathbf{k}\right)}=\mathcal{G}\left(x^{\prime}, x, \overline{\mathbf{k}}\right) .
$$

Свойства аналитичности функции Грина вытекают из свойств $\mathcal{G}_{1}(\mathbf{k})$ и нашего предположения о "малости" возмушения $u_{2}$. В частности, это означает, что $\mathcal{G}(\mathbf{k})$ аналитична в области $\mathbf{k}_{\Re} \mathbf{k}_{\Im} \neq 0$, где в силу (3.3) и (3.4) удовлетворяет уравнениям

$$
\frac{\partial \mathcal{G}(\mathbf{k})}{\partial \mathbf{k}_{\Re}}=\frac{\operatorname{sgn} \mathbf{k}_{\Im}}{2 \pi i} \Phi(\mathbf{k}) \otimes \Psi(\mathbf{k}), \quad \frac{\partial \mathcal{G}(\mathbf{k})}{\partial \mathbf{k}_{\Im}}=\frac{\operatorname{sgn} \mathbf{k}_{\Im}}{2 \pi} \Phi(\mathbf{k}) \otimes \Psi(\mathbf{k}) .
$$

Функция Грина обладает стандартным разрывом при $\mathbf{k}_{\Im}=0$ и дополнительным при $\mathbf{k}_{\Re}=0$, если $\left|\mathbf{k}_{\Im}\right|<\max _{j} \varkappa_{j}$. 
Для описания скачка на мнимой оси воспользуемся тождеством Гильберта (3.2), записывая его в силу (2.43), (3.11) с помошью функций Грина. Тогда благодаря $(2.44)$ мы получаем, что

$$
\begin{aligned}
\mathcal{G}(+0+ & \left.i \mathbf{k}_{\Im}\right)-\mathcal{G}\left(-0+i \mathbf{k}_{\Im}\right)=-\operatorname{sgn} \mathbf{k}_{\Im} \sum_{j} t_{j} \theta\left(\varkappa_{j}-\left|\mathbf{k}_{\Im}\right|\right) \times \\
& \times\left(\mathcal{G}\left( \pm 0+i \mathbf{k}_{\Im}\right) \overleftarrow{\mathcal{L}}_{1} \varphi\left(i \varkappa_{j}\right)\right) \otimes\left(\psi\left(i \varkappa_{j}\right) \overrightarrow{\mathcal{L}}_{1} \mathcal{G}\left(\mp 0+i \mathbf{k}_{\Im}\right)\right)
\end{aligned}
$$

где выражения в скобках понимаются в смысле (3.8). Равенство (3.15) предлагает введение функций $\Phi_{j}\left(x, \mathbf{k}_{\Im}\right)$ и $\Psi_{j}\left(x, \mathbf{k}_{\Im}\right)$ посредством соотношений

$$
\Phi_{j}\left(\mathbf{k}_{\Im}\right)=\mathcal{G}\left(+0+i \mathbf{k}_{\Im}\right) \overleftarrow{\mathcal{L}}_{1} \varphi\left(i \varkappa_{j}\right), \quad \Psi_{j}\left(\mathbf{k}_{\Im}\right)=\psi\left(i \varkappa_{j}\right) \overrightarrow{\mathcal{L}}_{1} \mathcal{G}\left(+0+i \mathbf{k}_{\Im}\right)
$$

Теперь из (3.15) (нижний знак) мы выводим для этого разрыва следующее выражение:

$$
\begin{aligned}
& \mathcal{G}\left(+0+i \mathbf{k}_{\Im}\right)-\mathcal{G}\left(-0+i \mathbf{k}_{\Im}\right)= \\
& \quad=-\sum_{l, m} \theta\left(\varkappa_{l}-\left|\mathbf{k}_{\Im}\right|\right) \Phi_{l}\left(\mathbf{k}_{\Im}\right)\left(A\left(\mathbf{k}_{\Im}\right)^{-1}\right)_{l m} \otimes \Psi_{m}\left(\mathbf{k}_{\Im}\right),
\end{aligned}
$$

где $A\left(\mathbf{k}_{\Im}\right)$ - матрища с элементами

$$
\begin{aligned}
A_{l m}\left(\mathbf{k}_{\Im}\right)= & \frac{\delta_{l m}}{t_{l} \operatorname{sgn} \mathbf{k}_{\Im}}-\theta\left(\min \left\{\varkappa_{l}, \varkappa_{m}\right\}-\left|\mathbf{k}_{\Im}\right|\right) \times \\
& \times\left(\psi\left(i \varkappa_{l}\right) \overrightarrow{\mathcal{L}}_{1} \mathcal{G}\left(+0+i \mathbf{k}_{\Im}\right) \overleftarrow{\mathcal{L}}_{1} \varphi\left(i \varkappa_{m}\right)\right)
\end{aligned}
$$

и где “математическое ожидание” (последний множитель в (3.18)) равно

$$
\int d x \int d x^{\prime} \psi\left(x, i \varkappa_{l}\right) \varphi\left(x^{\prime}, i \varkappa_{m}\right) \mathcal{L}_{1}\left(x, \partial_{x}\right) \mathcal{L}_{1}^{\mathrm{d}}\left(x^{\prime}, \partial_{x}^{\prime}\right) \mathcal{G}\left(x, x^{\prime},+0+i \mathbf{k}_{\Im}\right)
$$

(ср. обозначения (3.8)) и является функцией только от $\mathbf{k}_{\Im}$.

В предположении однозначной разрешимости интегрального уравнения для функции Грина матрица $A\left(\mathbf{k}_{\Im}\right)$ несингулярна, а в силу $(2.27)$ и (3.13) легко видеть, что

$$
A\left(\mathbf{k}_{\Im}\right)^{\dagger}=B A\left(-\mathbf{k}_{\Im}\right) B^{-1}
$$

где мы ввели диагональную матрицу $B=\operatorname{diag}\left\{b_{1}, \ldots, b_{N}\right\}$. Заметим, что $B$ - эрмитова матрица, поскольку $b_{j}$ в $(2.26)$ вешественны. 
3.3. Скачок решений Йоста на мнимой оси. Решения Йоста $\Phi(x, \mathbf{k})$ и $\Psi(x, \mathbf{k})$, введенные в (3.8), удовлетворяют в силу (3.12) интегральным уравнениям

$$
\Phi(\mathbf{k})=\varphi(\mathbf{k})+\mathcal{G}_{1}(\mathbf{k}) U_{2} \Phi(\mathbf{k}), \quad \Psi(\mathbf{k})=\psi(\mathbf{k})+\Psi(\mathbf{k}) U_{2} \mathcal{G}_{1}(\mathbf{k})
$$

причем благодаря (3.8) и (3.13)

$$
\overline{\Phi(x, \mathbf{k})}=\Psi(x, \overline{\mathbf{k}}) .
$$

В силу свойств функции Грина $\mathcal{G}(\mathbf{k})$ решения Йоста являются аналитическими функциями переменной $\mathbf{k} \in \mathbb{C}$, если $\mathbf{k}_{\Re} \mathbf{k}_{\Im} \neq 0$, и в ситуации обшего положения имеют стандартный разрьв на вешественной оси $\mathbf{k}_{\Im}=0$ и дополнительный разрыв на отрезке $\mathbf{k}_{\Re}=$ $0,\left|\mathbf{k}_{\Im}\right| \leqslant \max _{j} \varkappa_{j}$,мнимой оси. Принимая во внимание, что функции $\varphi(\mathbf{k})$ и $\psi(\mathbf{k})$ непрерывны при $\mathbf{k}_{\Re}=0$ и применяя к (3.15) операцию $\overleftarrow{\mathcal{L}}_{1} \varphi\left(i \mathbf{k}_{\Im}\right)$, из $(3.8)$ для этого скачка мы получаем

$$
\Phi\left(x,+0+i \mathbf{k}_{\Im}\right)-\Phi\left(x,-0+i \mathbf{k}_{\Im}\right)=\sum_{l} \theta\left(\varkappa_{l}-\left|\mathbf{k}_{\Im}\right|\right) \Phi_{l}\left(x, \mathbf{k}_{\Im}\right) w_{l}\left(\mathbf{k}_{\Im}\right),
$$

где мы ввели функции

$$
w_{l}\left(\mathbf{k}_{\Im}\right) \equiv t_{l} \operatorname{sgn} \mathbf{k}_{\Im}\left(\psi\left(i \varkappa_{l}\right) \overrightarrow{\mathcal{L}}_{1} \mathcal{G}\left(-0+i \mathbf{k}_{\Im}\right) \overleftarrow{\mathcal{L}}_{1} \varphi\left(i \mathbf{k}_{\Im}\right)\right)
$$

а выражения в скобках понимаются, как в (3.18). Функции $w_{l}\left(\mathbf{k}_{\Im}\right)$ являются спектральными данными, описываюшими скачок решения Йоста на мнимой оси. Аналогичное соотношение для дуального решения $\Psi(x, \mathbf{k})$ можно получить из свойств сопряжения $(3.21)$ и условий

$$
\overline{\Phi_{j}\left(x, \mathbf{k}_{\Im}\right)}=b_{j} \Psi_{j}\left(x,-\mathbf{k}_{\Im}\right), \quad \overline{\Psi_{j}\left(x, \mathbf{k}_{\Im}\right)}=\frac{\Phi_{j}\left(x,-\mathbf{k}_{\Im}\right)}{b_{j}},
$$

которые, в свою очередь, следуют из (2.27) и (3.13). Итак, по формуле (3.22) скачок решения Йоста на мнимой оси выражается через введенные в $(3.16)$ функции $\Phi_{l}\left(x, \mathbf{k}_{\Im}\right)$, $\Psi_{l}\left(x, \mathbf{k}_{\Im}\right)$. Мы доказываем, что они, как и решения Йоста, удовлетворяют дифференциальным уравнениям $\overrightarrow{\mathcal{L}} \Phi_{l}\left(\mathbf{k}_{\Im}\right)=\Psi_{l}\left(\mathbf{k}_{\Im}\right) \overleftarrow{\mathcal{L}}=0$. Эти функции являются обобшениями $\varphi\left(i \varkappa_{l}\right)$ и $\psi\left(i \varkappa_{l}\right)$ на случай $u_{2} \neq 0$. При этом в отличие от последних $\Phi_{l}\left(x, \mathbf{k}_{\Im}\right)$ и $\Psi_{l}\left(x, \mathbf{k}_{\Im}\right)$ не совпадают с решениями Йоста в некоторых точках и нетривиально зависят от $\mathbf{k}_{\Im}$. Учитьвая (3.15), замечаем, что эти функции требуются нам лишь на интервале $\left|\mathbf{k}_{\Im}\right|<\varkappa_{l}$. В дальнейшем мы называем их вспомогательными решениями Йоста. Как следует из свойств полной функции Грина, эти решения разрывны при $\mathbf{k}_{\Im}=0$. 


\section{4. Поведение функции Грина, решений Йоста и спектральных данных}

в точках $\pm i \varkappa_{j} . \mathrm{B}(2.38)$ было показано, что $\mathcal{G}_{1}(\mathbf{k})$ имеет логарифмические особенности во всех точках $\mathbf{k}= \pm i \varkappa_{j}, j=1, \ldots, N$. Рассмотрим, как эти особенности влияют на свойства полной функции Грина $\mathcal{G}(\mathbf{k})$ и построенных по ней объектов. Выберем некоторое $\varkappa_{j}$ и предположим, что $\mathbf{k}$ принадлежит таким окрестностям точек $\pm i \varkappa_{j}$, которые не включают других точек $\pm i \varkappa, l \neq j$, или точек на вещественной оси. Введем функцию

$$
\mathcal{G}_{1, j}(\mathbf{k})=\mathcal{G}_{1}(\mathbf{k})-\gamma_{j}(\mathbf{k}) \varphi(\mathbf{k}) \otimes \psi(\mathbf{k}),
$$

которая по формуле (2.38) конечна в указанных окрестностях точек $\pm i \varkappa_{j}$, но, возможно, разрывна в соответствии с (2.44). Этот недостаток регуляризации (3.25) по сравнению с регуляризацией $\mathcal{G}_{1, \mathrm{reg}}(\mathbf{k})$ в $(2.38)$ компенсируется тем фактом, что произведение $e^{i \ell(\mathbf{k})\left(x-x^{\prime}\right)} \mathcal{G}_{1, j}\left(x, x^{\prime}, \mathbf{k}\right)$ ограничено на $x$-плоскости. Определим теперь новую функцию Грина нестационарного уравнения Шредингера с потенциалом (1.3) посредством интегрального уравнения $\mathcal{G}_{j}(\mathbf{k})=\mathcal{G}_{1, j}(\mathbf{k})+\mathcal{G}_{1, j}(\mathbf{k}) U_{2} \mathcal{G}_{j}(\mathbf{k})$ или дуального к нему. Тогда $\mathcal{G}_{j}(\mathbf{k})$ - регуляризация $\mathcal{G}(\mathbf{k})$ в окрестностях $\pm i \varkappa_{j}$ такая, что

$$
\mathcal{G}(\mathbf{k})=\mathcal{G}_{j}(\mathbf{k})+\gamma_{j}(\mathbf{k}) \widetilde{\Phi}_{j}(\mathbf{k}) \otimes \Psi(\mathbf{k}),
$$

где мы используем обозначения (3.8) для решений Йоста и аналогично вводим

$$
\widetilde{\Phi}_{j}(\mathbf{k})=\mathcal{G}_{j}(\mathbf{k}) \overleftarrow{\mathcal{L}}_{1} \varphi(\mathbf{k}), \quad \widetilde{\Psi}_{j}(\mathbf{k})=\psi(\mathbf{k}) \overrightarrow{\mathcal{L}}_{1} \mathcal{G}_{j}(\mathbf{k})
$$

Функции $\widetilde{\Phi}_{j}(\mathbf{k})$ и $\widetilde{\Psi}_{j}(\mathbf{k})$ также являются решениями нестационарного уравнения Шредингера и дуального к нему, соответственно. Эти функции ограничены в окрестностях точек $\pm i \varkappa_{j}$, но могут быть разрывны в этих окрестностях. Те же свойства выполняются и для функций

$$
g_{j}(\mathbf{k})=\left(\psi(\mathbf{k}) \overrightarrow{\mathcal{L}}_{1} \mathcal{G}_{j}(\mathbf{k}) \overleftarrow{\mathcal{L}}_{1} \varphi(\mathbf{k})\right)
$$

где выражение в скобках понимается подобно "математическому ожиданию" в (3.18). Применяя $\psi(\mathbf{k}) \overrightarrow{\mathcal{L}}_{1 \text { к }}(3.26)$ слева, мы получаем по формулам $(3.8)$ и (3.27), (3.28), что $\Psi(\mathbf{k})=\widetilde{\Psi}(\mathbf{k})\left(1-\gamma_{j}(\mathbf{k}) g_{j}(\mathbf{k})\right)^{-1}$, так что $(3.26)$ может быть записано как

$$
\mathcal{G}(\mathbf{k})=\mathcal{G}_{j}(\mathbf{k})+\frac{\gamma_{j}(\mathbf{k})}{1-\gamma_{j}(\mathbf{k}) g_{j}(\mathbf{k})} \widetilde{\Phi}_{j}(\mathbf{k}) \otimes \widetilde{\Psi}_{j}(\mathbf{k})
$$

Теперь мы видим, что поведение функции Грина и решений Йоста в окрестностях точек $\pm i \varkappa_{j}$ определяется поведением функций $g_{j}(\mathbf{k})$. Если

$$
g_{j}(\mathbf{k})=o(1), \quad \mathbf{k} \sim \pm i \varkappa_{j},
$$

то функция Грина имеет логарифмические особенности в точках $\pm i \varkappa_{j}$, а решения Йоста ограничены, но разрывны в этих точках. С другой стороны, если

$$
g_{j}(\mathbf{k})=O(1), \quad \mathbf{k} \sim \pm i \varkappa_{j},
$$


и предел (также зависящий от пути предельного перехода) отличен от нуля, то

$$
\Phi(\mathbf{k})=o(1), \quad \Psi(\mathbf{k})=o(1), \quad \mathbf{k} \sim \pm i \varkappa_{j},
$$

более точно, в правых частях мы имеем порядок $1 / \ln \left(\left|\mathbf{k} \mp i \varkappa_{j}\right|\right)$.

В дальнейшем мы полагаем, что условие (3.31) выполняется для всех $j=1, \ldots, N$ (имеется только одно условие для каждого $j$, поскольку по формулам (2.20) и (3.13) $\left.\overline{g_{j}(\mathbf{k})}=g_{j}(\overline{\mathbf{k}})\right)$. В частности, при таком условии

$$
\Phi_{j}\left( \pm \varkappa_{j}\right)=\Psi_{j}\left( \pm \varkappa_{j}\right)=0, \quad j=1, \ldots, N,
$$

в то время как значения $\Phi_{m}\left( \pm \varkappa_{j}\right)$ и $\Psi_{m}\left( \pm \varkappa_{j}\right)$ вспомогательных решений для $m$ таких, что $\varkappa_{m}>\varkappa_{l}$, конечны и отличны от нуля, и

$$
\psi\left(i \varkappa_{j}\right) \overrightarrow{\mathcal{L}}_{1} \Phi_{m}\left( \pm \varkappa_{j}\right)=0, \quad \Psi_{m}\left( \pm \varkappa_{j}\right) \overleftarrow{\mathcal{L}}_{1 \varphi}\left(i \varkappa_{j}\right)=0
$$

При условии (3.31) матричные элементы $A_{l m}\left(\mathbf{k}_{\Im}\right)$ имеют конечные пределы при $\mathbf{k}_{\Im}=$ $\pm \varkappa_{j}$, как следует из (3.18). В частности, с учетом (3.27) и (3.34) получаем

$$
A\left( \pm \varkappa_{j}\right)_{j m}=A\left( \pm \varkappa_{j}\right)_{m j}=\frac{ \pm \delta_{j m}}{t_{j}}
$$

что совпадает со значениями $A_{j m}\left(\mathbf{k}_{\Im}\right)$ и $A_{m j}\left(\mathbf{k}_{\Im}\right)$ при $\mathbf{k}_{\Im}>\min \left\{\varkappa_{j}, \varkappa_{m}\right\}$. Наконец, используя (3.23), можно доказать, что при условии (3.31)

$$
w_{l}\left( \pm \varkappa_{j}\right)_{j m}=0 \text { для всех } j \text { и } l: \varkappa_{j} \leqslant \varkappa_{l} .
$$

Выведенные свойства решений Йоста и спектральных данных позволяют восстановить вспомогательные решения Йоста из граничных значений самих решений Йоста. Действительно, интегрируя (3.14) при $\mathbf{k}_{\Re}= \pm 0$ и используя обозначения (2.42) для предельного значения полной функции Грина на вешественной оси, мы получаем

$$
\mathcal{G}\left( \pm 0+i \mathbf{k}_{\Im}\right)=\mathcal{G}^{\sigma}( \pm 0)+\frac{\operatorname{sgn} \mathbf{k}_{\Im}}{2 \pi} \int_{0}^{\mathbf{k}_{\Im}} d \alpha t(i \alpha) \Phi( \pm 0+i \alpha) \otimes \Psi( \pm 0+i \alpha),
$$

где $\sigma=\operatorname{sgn} \mathbf{k}_{\Im}$. Тем же способом в силу равенств $(3.16),(3.18),(3.23)$ и (3.33) мы находим в области $\left|\mathbf{k}_{\Im}\right|<\varkappa_{j}$, что

$$
\Phi_{j}\left(\mathbf{k}_{\Im}\right)=\frac{\operatorname{sgn} \mathbf{k}_{\Im}}{2 \pi} \int_{\varkappa_{j} \operatorname{sgn} \mathbf{k}_{\Im}}^{\mathbf{k}_{\Im}} d \alpha t(i \alpha) \Phi(+0+i \alpha) \sum_{l} b_{l} \overline{w_{l}(-\alpha)} A_{l j}(\alpha) .
$$

Это выражение обобщает одномерные соотношения (2.27). Так же из равенств (3.14), $(3.18),(3.23)$ и (3.35) получаем

$$
\begin{aligned}
\left(A^{-1}\left(\mathbf{k}_{\Im}\right)\right)_{j m}= & t_{m} \operatorname{sgn} \mathbf{k}_{\Im} \delta_{j m}+\theta\left(\min \left\{\varkappa_{m}, \varkappa_{j}\right\}-\left|\mathbf{k}_{\Im}\right|\right) \frac{\operatorname{sgn} \mathbf{k}_{\Im}}{2 \pi} b_{m} \times \\
& \times \int_{\min \left\{\varkappa_{m}, \varkappa_{j}\right\} \operatorname{sgn} \mathbf{k}_{\Im}}^{\mathbf{k}_{\Im}} d \alpha t(i \alpha) w_{j}(\alpha) \overline{w_{m}(-\alpha)},
\end{aligned}
$$

что выражает спектральные данные $A_{m j}\left(\mathbf{k}_{\Im}\right)$ через $w_{j}\left(\mathbf{k}_{\Im}\right)$. Непосредственно проверяется, что описанные выше свойства решений Йоста и спектральных данных совместны с представлениями (3.38) и (3.39). 
3.5. Билинейное представление для резольвенты и соотношения между функциями Грина. Пусть $q_{1} \neq 0$. Производная $\widehat{M}(q)$ по $q_{2}$ при $q_{2} \neq 0$ дана в $(3.4)$, а скачок при $q_{2}=0$ следует из (3.11) и (3.17). Эти равенства позволяют нам восстановить хэт-ядро $\widehat{M}\left(x, x^{\prime} ; q\right)$ резольвенты с точностью до не зависящего от $q_{2}$ слагаемого. Это слагаемое фиксируется условием, что ядро $M\left(x, x^{\prime} ; q\right)$ (определенное по формуле (2.3)) принадлежит $\mathcal{S}^{\prime}\left(\mathbb{R}^{6}\right)$. Опуская детали этой конструкции (см. [7]), мы приведем здесь окончательное выражение:

$$
\begin{aligned}
\widehat{M}\left(x, x^{\prime} ; q\right)= & \frac{1}{2 \pi i} \int_{\mathbf{k}_{\Im}=q_{1}} d \mathbf{k}_{\Re}\left[\theta\left(x_{2}-x_{2}^{\prime}\right)-\theta\left(2 \mathbf{k}_{\Re} \mathbf{k}_{\Im}-q_{2}\right)\right] t(\mathbf{k}) \Phi(x, \mathbf{k}) \Psi\left(x^{\prime}, \mathbf{k}\right)- \\
& -\operatorname{sgn} q_{1}\left[\theta\left(x_{2}-x_{2}^{\prime}\right)-\theta\left(-q_{2}\right)\right] \sum_{l, m} \theta\left(\min \left\{\varkappa_{l}, \varkappa_{m}\right\}-\left|q_{1}\right|\right)\left(A\left(q_{1}\right)^{-1}\right)_{l m} \times \\
& \times \Phi_{l}\left(x, q_{1}\right) \Psi_{m}\left(x^{\prime}, q_{1}\right)
\end{aligned}
$$

которое дает билинейное представление резольвенты в терминах решений Йоста. Заметим, что в силу ограниченности ядра $M\left(x, x^{\prime} ; q\right)$ по $q$ и его непрерывности по $q_{1}$ при $q_{2} \neq 0$ представление (3.40) справедливо при всех $q$.

Это билинейное представление для резольвенты приводит с учетом (3.7) к следующему билинейному представлению для функции Грина:

$$
\begin{aligned}
\mathcal{G}\left(x, x^{\prime}, \mathbf{k}\right)= & \int \frac{d k^{\prime}}{2 \pi i}\left[\theta\left(x_{2}-x_{2}^{\prime}\right)-\theta\left(\mathbf{k}_{\Im} k^{\prime}\right)\right] t\left(k^{\prime}+\mathbf{k}\right) \Phi\left(x, k^{\prime}+\mathbf{k}\right) \Psi\left(x^{\prime}, k^{\prime}+\mathbf{k}\right)- \\
& -\operatorname{sgn} \mathbf{k}_{\Im}\left[\theta\left(x_{2}-x_{2}^{\prime}\right)-\theta\left(-\mathbf{k}_{\Re} \mathbf{k}_{\Im}\right)\right] \times \\
& \times \sum_{l, m} \theta\left(\min \left\{\varkappa_{l}, \varkappa_{m}\right\}-\left|\mathbf{k}_{\Im}\right|\right)\left(A\left(\mathbf{k}_{\Im}\right)^{-1}\right)_{l m} \Phi_{l}\left(x, \mathbf{k}_{\Im}\right) \Psi_{m}\left(x^{\prime}, \mathbf{k}_{\Im}\right),
\end{aligned}
$$

обобшающему (2.35). Более того, благодаря (3.10) билинейное представление (3.40) дает представление для опережающей и запаздывающей функций Грина также в терминах решений Йоста:

$$
\begin{aligned}
\mathcal{G}_{ \pm}\left(x, x^{\prime}\right)= & \pm \\
& \theta\left( \pm\left(x_{2}-x_{2}^{\prime}\right)\right)\left(\frac{1}{2 \pi i} \int d k t^{\sigma}(k) \Phi^{\sigma}(x, k) \Psi^{\sigma}\left(x^{\prime}, k\right)-\right. \\
& \left.-\sigma \sum_{l, m}\left(A^{\sigma}\right)_{l m}^{-1} \Phi_{l}^{\sigma}(x) \Psi_{m}^{\sigma}\left(x^{\prime}\right)\right)
\end{aligned}
$$

где $\sigma=+,-$, и по аналогии с (2.42) мы ввели обозначения $(k \in \mathbb{R})$

$$
\begin{aligned}
\Phi^{ \pm}(x, k) & =\lim _{\mathbf{k}_{\Im} \rightarrow \pm 0} \Phi\left(x, k+i \mathbf{k}_{\Im}\right), & \Psi^{ \pm}(x) & =\lim _{\mathbf{k}_{\Im} \rightarrow \pm 0} \Psi\left(x, k+i \mathbf{k}_{\Im}\right), \\
\Phi_{l}^{ \pm}(x) & =\lim _{\mathbf{k}_{\Im} \rightarrow \pm 0} \Phi_{l}\left(x, \mathbf{k}_{\Im}\right), & \Psi_{l}^{ \pm}(x) & =\lim _{\mathbf{k}_{\Im} \rightarrow \pm 0} \Psi_{l}\left(x, \mathbf{k}_{\Im}\right)
\end{aligned}
$$

для значений решений Йоста на вешественной оси. Благодаря (3.21) и (3.24) эти предельные значения удовлетворяют следующим свойствам сопряжения:

$$
\overline{\Phi^{ \pm}(x, k)}=\Psi^{\mp}(x, k), \quad \overline{\Phi_{l}^{ \pm}(x)}=b_{l} \Psi_{l}^{\mp}(x), \quad k \in \mathbb{R}, \quad l=1, \ldots, N .
$$


Отметим здесь без доказательства, что левая часть равенства (3.42) не зависит от $\sigma$.

Непосредственно проверяется, что функции $\mathcal{G}_{ \pm}$удовлетворяют диффференциальным уравнениям $\overrightarrow{\mathcal{L}} \mathcal{G}_{ \pm}=\mathcal{G}_{ \pm} \overleftarrow{\mathcal{L}}=I$, свойству сопряжения

$$
\overline{\mathcal{G}_{ \pm}\left(x, x^{\prime}\right)}=\mathcal{G}_{\mp}\left(x^{\prime}, x\right)
$$

и интегральным уравнениям $\mathcal{G}_{ \pm}=\mathcal{G}_{1, \pm}+\mathcal{G}_{1, \pm} U_{2} \mathcal{G}_{ \pm}$и дуальным к ним.

Обычные и вспомогательные опережаюшие/запаздываюшие решения определяются (ср. с (3.8), (3.16)) как

$$
\begin{array}{ll}
\Phi_{ \pm}(k)=\mathcal{G}_{ \pm} \overleftarrow{\mathcal{L}}_{1} \varphi_{ \pm}(k), & \Phi_{ \pm, j}=\mathcal{G}_{ \pm} \overleftarrow{\mathcal{L}}_{1} \varphi\left(i \varkappa_{j}\right) \\
\Psi_{ \pm}(k)=\psi_{ \pm}(k) \overrightarrow{\mathcal{L}}_{1} \mathcal{G}_{ \pm}, & \Psi_{ \pm, j}=\psi\left(i \varkappa_{j}\right) \overrightarrow{\mathcal{L}}_{1} \mathcal{G}_{ \pm}
\end{array}
$$

Эти функции удовлетворяют нестационарному уравнению Шредингера с потенциалом $u$ и свойствам сопряжения

$$
\overline{\Phi_{ \pm}(x, k)}=\Psi_{\mp}(x, k), \quad \overline{\Phi_{ \pm, j}}=b_{j} \Psi_{\mp, j} .
$$

Предельные значения функции Грина на вещественной оси, как следует из (3.7), также связаны с пределами $M(q)$ при $q \rightarrow 0$, отличными от (3.10). Для этих предельных значений мы используем обозначения $\mathcal{G}^{ \pm}(k)$, как в $(2.42)$. Поскольку правые части равенств (3.41) и (3.42) выражены через решения Йоста, то, переходя в (3.41) к пределу $\mathbf{k} \rightarrow k+i \sigma 0$, по формулам (3.43) и (3.44) получаем

$$
\begin{aligned}
\mathcal{G}^{\sigma}(k)-\mathcal{G}_{ \pm} & =\frac{\mp 1}{2 \pi i} \int d k^{\prime} \theta\left( \pm \sigma\left(k^{\prime}-k\right)\right) t^{\sigma}\left(k^{\prime}\right) \Phi^{\sigma}\left(k^{\prime}\right) \otimes \Psi^{\sigma}\left(k^{\prime}\right) \pm \\
& \pm \sigma \theta(\mp \sigma k) \sum_{l, m}\left(A^{\sigma}\right)_{l m}^{-1} \Phi_{l}^{\sigma} \otimes \Psi_{m}^{\sigma} .
\end{aligned}
$$

Другое выражение для этой разности следует из тождества Гильберта (3.2). Для этого мы используем (3.7) (снова в пределе $\mathbf{k} \rightarrow k+i \sigma 0)$ и соотношения $(3.14),(2.49)$ и (2.50). Тогда

$$
\begin{aligned}
\mathcal{G}^{\sigma}(k)-\mathcal{G}_{ \pm} & =\frac{\mp 1}{2 \pi i} \int d \alpha \theta( \pm \sigma(\alpha-k)) \int d p \Phi_{ \pm}(p) r_{ \pm}^{\sigma}(p, \alpha) \otimes \psi^{\sigma}(\alpha) \overrightarrow{\mathcal{L}}_{1} \mathcal{G}^{\sigma}(k) \pm \\
& \pm \theta(\mp \sigma k) \sum_{j} t_{j} \Phi_{ \pm, j} \otimes \psi\left(i \varkappa_{j}\right) \overrightarrow{\mathcal{L}}_{1} \mathcal{G}^{\sigma}(k)
\end{aligned}
$$

где $r_{ \pm}^{\sigma}$ определено в (2.51).

3 Теоретическая и математическая физика, т. 144, № 2, 2005 г. 
3.6. Спектральные данные. В последнем равенстве разность функций Грина определяется через опережающие и запаздывающие решения, что непосредственно приводит к выражению решения Йоста через опережаюшее и запаздываюшее решения. Действительно, в силу определений (3.8), (3.16) (в предельном случае $(3.43),(3.44))$ и равенств (3.47), (3.48) мы получаем

$$
\begin{gathered}
t^{\sigma}(k) \Phi^{\sigma}(k)=\int d p \Phi_{ \pm}(p) \mathcal{R}_{ \pm}^{\sigma}(p, k)+\sum_{j} \Phi_{ \pm, j} \mathcal{R}_{ \pm, j}^{\sigma}(k), \quad \sigma=+,- \\
\Phi_{m}^{\sigma}=\int d p \Phi_{ \pm}(p) \widehat{\mathcal{R}}_{ \pm, m}^{\sigma}(p)+\theta( \pm \sigma) \Phi_{ \pm, m} \mp \theta(\mp \sigma) \sum_{j} t_{j} \Phi_{ \pm, j} A_{j m}^{\sigma}
\end{gathered}
$$

где в последней строке мы воспользовались (3.18) в пределе $\mathbf{k}_{\Im} \rightarrow \sigma 0$. Кроме того, мы ввели спектральные данные $\mathcal{R}_{ \pm}^{\sigma}, \mathcal{R}_{ \pm, j}^{\sigma}$ и $\widehat{\mathcal{R}}_{ \pm, m}^{\sigma}$ как

$$
\mathcal{R}_{ \pm}^{\sigma}(p, k)=\int d \alpha r_{ \pm}^{\sigma}(p, \alpha) R_{ \pm}^{\sigma}(\alpha, k),
$$

где $R_{ \pm}^{\sigma}$ - треугольньй оператор, $R_{ \pm}^{\sigma}(p, k)=\delta(p-k) \mp \theta( \pm \sigma(p-k)) R^{\sigma}(p, k)$,

$$
R^{\sigma}(p, k)=\frac{t^{\sigma}(k)}{2 \pi i}\left(\psi^{\sigma}(p) \overrightarrow{\mathcal{L}}_{1} \mathcal{G}^{\sigma}(k) \overleftarrow{\mathcal{L}}_{1} \varphi^{\sigma}(k)\right)
$$

и выражение в скобках снова понимается, как в формуле (3.18). Далее, $\mathcal{R}_{ \pm, j}^{\sigma}(k)=$ $\pm \theta(\mp \sigma k) \mathcal{R}_{j}^{\sigma}(k)$, где

$$
\mathcal{R}_{j}^{\sigma}(k)=t_{j} t^{\sigma}(k)\left(\psi\left(i \varkappa_{j}\right) \overrightarrow{\mathcal{L}}_{1} \mathcal{G}^{\sigma}(k) \overleftarrow{\mathcal{L}}_{1} \varphi^{\sigma}(k)\right)
$$

$$
\widehat{\mathcal{R}}_{ \pm, m}^{\sigma}(p)=\int d \alpha r_{ \pm}^{\sigma}(p, \alpha) \widehat{R}_{ \pm, m}^{\sigma}(\alpha)
$$

где $\widehat{R}_{ \pm, m}^{\sigma}(k)=\mp \theta( \pm \sigma k) \widehat{R}_{m}^{\sigma}(k)$

$$
\widehat{R}_{m}^{\sigma}(k)=\frac{1}{2 \pi i}\left(\psi^{\sigma}(k) \overrightarrow{\mathcal{L}}_{1} \mathcal{G}^{\sigma}(+0) \overleftarrow{\mathcal{L}}_{1} \varphi^{\sigma}\left(i \varkappa_{m}\right)\right)
$$

Обратные соотношения, которые выражают опережающее и запаздывающее решения через решение Йоста, получаются аналогично, но путем редукции равенства (3.50). Благодаря введенным выше определениям спектральных данных мы находим

$$
\begin{gathered}
\Phi_{ \pm}(k)=\int d k^{\prime} \Phi^{\sigma}\left(k^{\prime}\right) \overline{\mathcal{R}_{ \pm}^{-\sigma}\left(k, k^{\prime}\right)}-2 \pi i \sigma \sum_{l, m} \Phi_{l}^{\sigma}\left(A^{\sigma}\right)_{l m}^{-1} \frac{1}{b_{m}} \overline{\widehat{\mathcal{R}}_{ \pm, m}^{-\sigma}(k)}, \\
\Phi_{ \pm, j}=\frac{-b_{j}}{2 \pi i t_{j}} \int d k^{\prime} \Phi^{\sigma}\left(k^{\prime}\right) \overline{\mathcal{R}_{ \pm, j}^{-\sigma}\left(k^{\prime}\right)}+\theta( \pm \sigma) \Phi_{j}^{\sigma} \mp \frac{\theta(\mp \sigma)}{t_{j}} \sum_{l} \Phi_{l}^{\sigma}\left(A^{\sigma}\right)_{l j}^{-1} .
\end{gathered}
$$


Наконец, подставляя эти равенства в (3.52), (3.53), мы получаем соотношения между предельными значениями решения Йоста на вешественной оси:

$$
\begin{aligned}
t^{\sigma}(k) \Phi^{\sigma}(k) & =\int d p \Phi^{-\sigma}(p) \mathcal{F}^{-\sigma}(p, k)+2 \pi i \sigma \sum_{l, m} \Phi_{l}^{-\sigma}\left(A^{-\sigma}\right)_{l m}^{-1} \frac{1}{b_{m}} \mathcal{F}_{m}^{-\sigma}(k), \\
\Phi_{j}^{\sigma} & =\int d p \Phi^{-\sigma}(p) \widehat{\mathcal{F}}_{j}^{-\sigma}(p)+2 \pi i \sigma \sum_{l, m} \Phi_{l}^{-\sigma}\left(A^{-\sigma}\right)_{l m}^{-1} \frac{1}{b_{m}} \mathcal{F}_{m j}^{-\sigma},
\end{aligned}
$$

обобшаюшие (2.53). Здесь по аналогии с (2.54) мы ввели альтернативные спектральные данные

$$
\begin{aligned}
\mathcal{F}^{-\sigma}\left(k, k^{\prime}\right)= & \int d k^{\prime \prime} \overline{\mathcal{R}_{ \pm}^{\sigma}\left(k^{\prime \prime}, k\right)} \mathcal{R}_{ \pm}^{\sigma}\left(k^{\prime \prime}, k^{\prime}\right)-\sum_{j} \frac{b_{j}}{2 \pi i t_{j}} \overline{\mathcal{R}_{ \pm, j}^{\sigma}(k)} \mathcal{R}_{ \pm, j}^{\sigma}\left(k^{\prime}\right), \\
\mathcal{F}_{l}^{-\sigma}\left(k^{\prime}\right)=- & \frac{\theta( \pm \sigma) b_{l}}{2 \pi i t_{l}} \mathcal{R}_{ \pm, l}^{\sigma}(k) \pm \frac{\theta(\mp \sigma)}{2 \pi i} \sum_{m=1}^{N} b_{l} A_{l m}^{-\sigma} \mathcal{R}_{ \pm, m}^{\sigma}(k)+ \\
& +\int d k^{\prime \prime} \overline{\widehat{\mathcal{R}}_{ \pm, l}^{\sigma}\left(k^{\prime \prime}\right)} \mathcal{R}_{ \pm}^{\sigma}\left(k^{\prime \prime}, k^{\prime}\right), \\
\widehat{\mathcal{F}}_{j}^{-\sigma}(k)=- & \frac{\theta( \pm \sigma) b_{j}}{2 \pi i t_{j}} \overline{\mathcal{R}_{ \pm, j}^{\sigma}(k)} \pm \sum_{l} \frac{b_{l} \theta(\mp \sigma)}{2 \pi i} A_{l j}^{\sigma} \overline{\overline{\mathcal{R}}_{ \pm, l}^{\sigma}(k)}+ \\
& +\int d k^{\prime} \overline{\mathcal{R}_{ \pm}^{\sigma}\left(k^{\prime}, k\right)} \widehat{\mathcal{R}}_{ \pm, j}^{\sigma}\left(k^{\prime}\right), \\
\mathcal{F}_{l j}^{-\sigma}= & -\frac{\theta( \pm \sigma) b_{l}}{2 \pi i t_{l}} \delta_{l j}+\frac{\theta(\mp \sigma)}{2 \pi i} \sum_{m=0}^{N} b_{l} A_{l m}^{-\sigma} t_{m} A_{m j}^{\sigma}+ \\
& +\int d k^{\prime} \overline{\widehat{\mathcal{R}}_{ \pm, l}^{\sigma}\left(k^{\prime}\right)} \widehat{\mathcal{R}}_{ \pm, j}^{\sigma}\left(k^{\prime}\right) .
\end{aligned}
$$

\section{4. ЗАКЛЮЧЕНИЕ}

Одним из преимушеств резольвентного подхода является существование билинейного представления (3.40), которое выражает расширенную резольвенту через решения Йоста. Сами эти решения, как и любые другие имеюшие отношение к делу решения рассматриваемой задачи рассеяния и дуальной к ней, получаются как специальные редукции соответствуюших функций Грина. Функции Грина, в свою очередь, являются значениями резольвенты в некоторых специальных точках. Это свойство дает нам простой и регулярный способ вывода соотношений между различными типами решений линейной задачи, а также между спектральными данными. Свойства обоих наборов ((3.54-(3.58)) и (3.63)-(3.66)) спектральных данных заслуживают специального рассмотрения. То же самое справедливо и для характеристических уравнений для них, которые следуют из комбинации равенств (3.52), (3.53) и (3.61), (3.62), а также из независимости левой части равенств (3.63)-(3.66) от знаков \pm . Эти соотношения, как и формулировка обратной задачи, основанной на полученных выше результатах, будут даны в [7]. 
Благодарности. Данная работа выполнена при частичной поддержке PRIN 2002 ‘Sintesi', РФФИ (грант № 02-01-00484), гранта НШ 2052.2003.1 и программы РАН “Математические методы нелинейной динамики". А. К. Погребков благодарит своих коллег по Факультету физики Университета г. Лечче за гостеприимство.

\section{Список литературы}

[1] Б. Б. Кадомчев, В. И. Петвиашвили. ДАН СССР. 1970. Т. 192. С. 753.

[2] В. Е. Захаров, А. Б. Шабат. Функц. анализ и его прилож. 1974. Т. 8. С. 43; В. С. Дрюма. Письма в ЖЭТФ. 1973. Т. 19. С. 219.

[3] V. E. Zakharov, S. V. Manakov. Sov. Sci. Rev.-Phys. Rev. 1979. V. 1. P. 133; S. V. Manakov. Physica. D. 1981. V. 3. P. 420; A. S. Fokas, M. J. Ablowitz. Stud. Appl. Math. 1983. V. 69. P. 211; M. Boiti, J. Léon, F. Pempinelli. Phys. Lett. A. 1989. V. 141. P. 96; Xin Zhou. Commun. Math. Phys. 1990. V. 128. P. 551; A. S. Fokas, L. Y. Sung. Math. Proc. Cambridge Philos. Soc. 1999. V. 125. P. 113.

[4] М. Бойти, Ф. Пемпинелли, А. К. Погребков, М. К. Поливанов. ТМФ. 1992. Т. 93. C. 181; M. Boiti, F. Pempinelli, A. K. Pogrebkov, M. C. Polivanov. Inverse Problems. 1992. V. 8. P. 331; M. Boiti, F. Pempinelli, A. Pogrebkov. Inverse Problems. 1994. V. 10. P. 505; J. Math. Phys. 1994. V. 35. Р. 4683; М. Бойти, Ф. Пемпинелли, А. К. Погребков. ТМФ. 1994. T. 99. C. 185; M. Boiti, F. Pempinelli, A. Pogrebkov. Spectral theory of solitons on a generic background for the KPI equation. In: Nonlinear Physics. Theory and Experiment. Proc. of the Workshop (Lecce, Italy, 29 June -7 July, 1995). Eds. E. Alfinito, M. Boiti, L. Martina, F. Pempinelli. Singapore: World Scientific, 1996. P. 37; Inverse Problems. 1997. V. 13. Р. L7; М. Бойти, Ф. Пемпинелли, А. К. Погребков, Б. Принари. ТМФ. 1998. T. 116. C. 3 .

[5] A. S. Fokas, A. K. Pogrebkov. Nonlinearity. 2003. V. 16. P. 771.

[6] M. Boiti, F. Pempinelli, A. K. Pogrebkov, B. Prinari. J. Math. Phys. 2003. V. 44. P. 3309.

[7] М. Бойти, Ф. Пемпинелли, А. К. Погребков, Б. Принари. Тр. МИАН. 2005 (в печати).

[8] В. Е. Захаров, С. В. Манаков, С. П. Новиков, Л. П. Питаевский. Теория солитонов. Метод обратной задачи. М.: Наука, 1980; Ф. Калоджеро, А. Дегасперис. Спектральные преобразования и солитоны. Методы решения и исследования нелинейных эволюционных уравнений. М.: Мир, 1982. 